EPJ Web of Conferences 49, 17006 (2013)

DOI: $10.1051 /$ epjconf/20134917006

(C) Owned by the authors, published by EDP Sciences, 2013

\title{
Top quark property at Tevatron
}

\author{
Hyun Su Lee ${ }^{1, a}$ on behalf of the CDF and D0 Collaborations \\ ${ }^{1}$ Department of Physics, Ewha Womans University, Seoul 120-750, Korea
}

\begin{abstract}
The top quark, the heaviest known elementary particle, remains by far the most interesting particle to test standard model as well as search new physics beyond standard model because of its large mass and unique properties. Having data collected about $10 \mathrm{fb}^{-1}$ of integrated luminosity of $p \bar{p}$ collisions, both experiments have been studied the top quark in all the possible directions. In this report, we present the recent measurements of the top quark properties from Tevatron including the mass, width, and $\mathrm{W}$ boson helicity using $t \bar{t}$ signature.
\end{abstract}

\section{Introduction}

The top quark, observed by both the CDF and D0 experiments in 1995 [1], is by far the heaviest known elementary particle and its mass is almost 40 times heavier than its isospin partner, the bottom $(b)$ quark [2]. Due to the heavy mass, the top quark plays an important role in electroweak radiative corrections relating the top quark mass $\left(\mathrm{M}_{\mathrm{top}}\right)$ and the $W$ boson mass to the mass of the predicted Higgs boson [3, 4]. The lifetime of the top quark is about 20 times shorter than the timescale for strong interactions, and therefore it does not form hadrons, giving us a unique opportunity to study a "bare" quark.

Top quarks at the Tevatron are predominantly produced in pairs, and decay almost always to a $W$ boson and a $b$ quark in the standard model (SM). The topology of $t \bar{t}$ events depends on the different decay of the two $W$ bosons. In the dilepton channel, each $W$ boson decay to charged lepton (electron and muon) and neutrino. Events in this channel thus contain two leptons, two $b$-quark jets, and two undetected neutrinos. Because of the presence of two leptons, this channel has the lowest background. However the dilepton channel has the smallest branching fraction. In the all-jets channel, each $W$ boson decays to two jets so that this channel contains two $b$ quark jets and four light quark jets. This channel has the largest branching fraction but also the largest background from QCD multijet production. The lepton+jets channel has one $W$ boson decaying leptonically and the other hadronically so that we have one charged lepton, two $b$-quark jets, two light quark jets, and one undetected neutrino. Because of the relatively large branching fraction with manageable backgrounds, lepton+jets channel is considered as the "golden channel" in the top quark studies. By this reason, the most results presented here use the lepton+jets final state.

\section{Measurements}

\subsection{Top quark mass}

The mass of the top quark is very important to estimate the SM Higgs boson because precise top and $W$ boson masses measurements can predict the mass of the Higgs boson either SM or beyond SM. Since the discovery of the top quark, both the CDF and D0 experiments have been improving the precision of the $\mathrm{M}_{\text {top }}$ measurement [5]. With up to $5.6 \mathrm{fb}^{-1}$ data, we obtain $\mathrm{M}_{\text {top }}=$ $173.18 \pm 0.94 \mathrm{GeV} / c^{2}$ with the combination of the Tevatron measurements as one can see in Fig. 1. The precision, $\Delta \mathrm{M}_{\text {top }} / \mathrm{M}_{\text {top }} \sim 0.6 \%$, is already surpassed the prediction of Run II experiments and reach to less than $1 \mathrm{GeV} / c^{2}$. With over $10 \mathrm{fb}^{-1}$ data in each experiment, we have performed the extensive studies and updated the measurements with improved precision.

To date the most precise measurement has been performed by CDF collaboration using lepton+jets channel with the full data set corresponding to $8.7 \mathrm{fb}^{-1} p \bar{p}$ collisions. We build templates of the reconstructed masses of the top quark and the hadronically decaying $W$ boson. In this measurement, we use not only larger samples but also improve jet energy calibration using an artificial neural network to achieve better jet energy resolution, and increase signal acceptance allowing less pure signal samples into the analysis. We found $\mathrm{M}_{\text {top }}=$ $172.85 \pm 1.11 \mathrm{GeV} / c^{2}$ [6]. D0 carried out the precise $\mathrm{M}_{\mathrm{top}}$ measurement in the dilepton channel. In this measurement, we used the results of jet energy scale measurement in the lepton+jets channel. In this way, we significantly reduced overall jet energy scale uncertainty in the dilepton final states. We found $\mathrm{M}_{\text {top }}=173.9 \pm 2.5 \mathrm{GeV} / c^{2}$ [7]. CDF have another full data set measurement in the different decay topology of jets with large missing transverse energy. In this measurement, we veto the events having reconstructed lepton so that the selected events are orthogonal to the lepton+jets channel. We perform event recon-

\footnotetext{
ae-mail: hyunsulee@ewha.ac.kr
} 


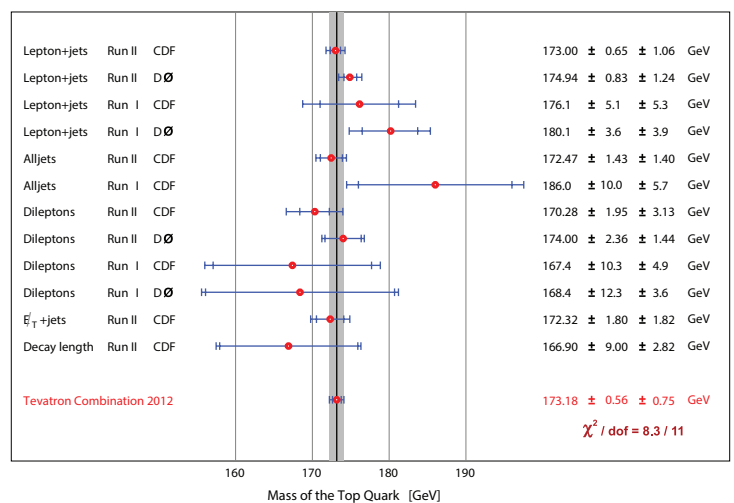

Figure 1. Summary of the Tevatron top quark mass measurements and its combination with data up to $5.6 \mathrm{fb}^{-1}$.

struction by assuming the missing particle as leptonic $W$ boson. Using templates of the reconstructed masses of the top quark mass and the hadronically decaying $W$ boson, we obtain $\mathrm{M}_{\text {top }}=173.9 \pm 1.9 \mathrm{GeV} / c^{2}$ [8]. Even though all the new measurements have significant improvements in the precisions compared with previous measurements, these are not included in the recent Tevatron average yet. Therefore, we expect much better precision with new combination at the Tevatron as well as the world averages.

There are different definitions of quark masses in the theoretical framework [9]. M $\mathrm{M}_{\text {top }}$ of the $\overline{\mathrm{MS}}$ renormalization scheme, $M_{\overline{\mathrm{MS}}}$, differs from the pole mass, $M_{\text {ploe }}$, by about $10 \mathrm{GeV} / c^{2}[9,10]$. The direct $\mathrm{M}_{\text {top }}$ measurements are all calibrated with MC simulations; the measured quantities therefore correspond to the $\mathrm{M}_{\text {top }}$ scheme used in the MC simulations, $M_{\mathrm{MC}}$. Even though it is usually assumed that $M_{\mathrm{MC}}$ is the same as $M_{\text {pole }}$ [5], there are a number of theoretical questions in relating $M_{\text {pole }}$ to $M_{\mathrm{MC}}[9,11]$. D0 collaboration extract the $M_{\text {ploe }}$, and the $M_{\overline{\mathrm{MS}}}$, comparing the measured inclusive $t \bar{t}$ production cross section with fully inclusive calculations at higherorder QCD. This extraction provides an important test of the mass scheme as applied in MC simulations and gives complementary information. Depending on the different theoretical calculations, we obtain $\mathrm{M}_{\text {top }}$ using this method consistent with but slight lower than $\mathrm{M}_{\text {top }}$ from direct measurements [12].

\subsection{Top and anti-top quark mass difference}

The precision determination of $\mathrm{M}_{\mathrm{top}}$ allows us to measure the mass difference between top quark and anti-top quark to a few $\mathrm{GeV}$. In the CPT theorem, which is fundamental to any local Lorentz-invariant quantum field theory, the quark mass should be same as the mass of its anti-quark partner. Despite the fact that no violations have ever been observed in the meson and baryon sectors, it is important to test CPT violation in all possible sectors such as quarks and high mass particles.

D0 collaboration has direct measurements of the top quark and the anti-top quark mass difference $\left(\delta \mathrm{M}_{\text {top }}\right.$
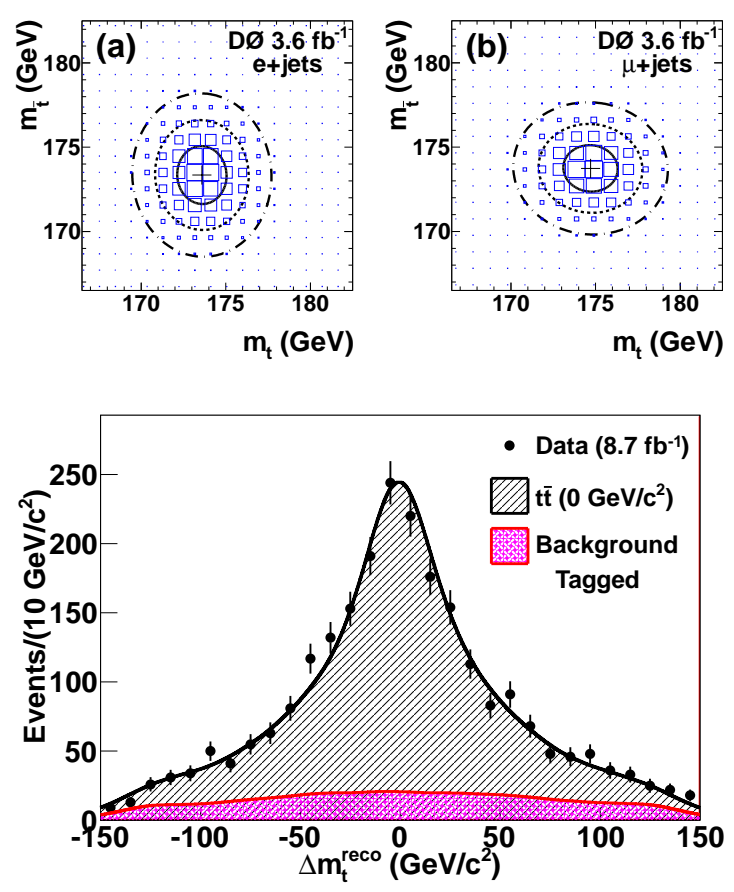

Figure 2. Up: Two-dimensional likelihood densities in top quark mass $\left(m_{t}\right)$ and anti-top quark mass $\left(m_{\bar{t}}\right)$ for the (a) e+jets and (b) $\mu+$ jets channels. The contours represent the 1,2 , and 3 standard deviations from $3.6 \mathrm{fb}^{-1} \mathrm{D} 0$ data. Down: Distributions of the reconstructed mass difference $\left(m_{t}-m_{\bar{t}}\right)$ for $b$-tagged events. The data from CDF $8.7 \mathrm{fb}^{-1}$ are overlaid with the prediction of SM.

) in the lepton+jets channel using the matrix element method [13]. In the matrix element calculation, one assumes SM-like $t \bar{t}$ production and decay, where identical particle and antiparticle masses are assumed for $b$ quarks and $\mathrm{W}$ bosons but not for top quarks. The likelihood contours of the data fit with $3.6 \mathrm{fb}^{-1}$ of $p \bar{p}$ collisions are shown in Fig. 2 (up) in the electron and muon channels. We measure $\delta \mathrm{M}_{\text {top }}=0.8 \pm 1.9 \mathrm{GeV} / c^{2}$ [13]. CDF collaboration has a measurement using the template method [14]. We reconstruct the mass difference using modified kinematic fitter allowing mass difference between hadronic top quark and leptonic top quark. Using full data set of $8.7 \mathrm{fb}^{-1} p \bar{p}$ collisions, we measure $\delta \mathrm{M}_{\text {top }}=-1.95 \pm 1.26 \mathrm{GeV} / c^{2}$ [14]. The data distribution of the reconstructed mass difference compared with the SM prediction is shown in Fig. 2 (down). All measurements are consistent with no mass difference and conservation of CPT symmetry.

\subsection{Top quark width}

Because of large mass, the top quark have the largest decay width and hence the shortest lifetime. Its short lifetime, which is fater than the hadronization time scale, make a unique opportunity to study bare quark [2]. By measuring the total decay width ( $\left.\Gamma_{\text {top }}\right)$, we can calculate the lifetime as the inverse of the width and prove whether the top quark decay before hadronization. The standard model prediction of $\Gamma_{\text {top }}$ in the next leading order is about $1.3 \mathrm{GeV}$ at $\mathrm{M}_{\mathrm{top}}=172.5 \mathrm{GeV} / c^{2}$ with ap- 


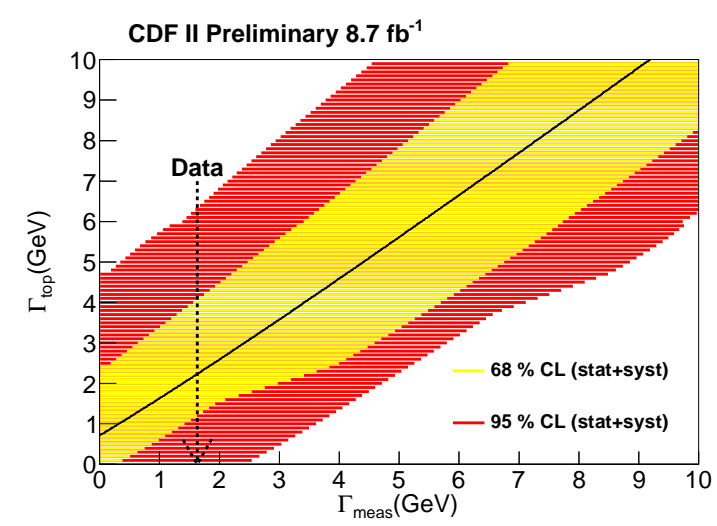

Figure 3. The confidence bands of $\Gamma_{\text {top }}$ for a $68 \%$ and $95 \%$ are shown together with the most probable value (thin solid line). The measured value from the data $(1.66 \mathrm{GeV})$ is indicated by an arrow.

proximately $1 \%$ precision. A deviation from the SM prediction, therefore, could indicate a significant contribution from non-SM physics processes. CDF collaboration has a direct measurement of the top quark width ( $\left.\Gamma_{\text {top }}\right)$ using the full data set of $8.7 \mathrm{fb}^{-1} p \bar{p}$ collisions. The $\mathrm{M}_{\text {top }}$ and the mass of $\mathrm{W}$ boson that decays hadronically are reconstructed for each event and compared with templates of different $\Gamma_{\text {top }}$ and deviations from nominal jet energy scale $\left(\Delta_{\mathrm{JES}}\right)$ to perform a simultaneous fit for both parameters, where $\Delta_{\mathrm{JES}}$ is used for the in situ calibration of the jet energy scale. By applying a Feldman-Cousins approach [15], we obtain $\Gamma_{\text {top }}=2.21_{-1.11}^{+1.84} \mathrm{GeV}$ as one can see in Fig. 3 [16]. We also estabilish a $95 \%$ confidence level upper limit of $\Gamma_{\text {top }}<6.38 \mathrm{GeV}$. D0 collaboration has an indirect determination of $\Gamma_{\text {top }}$ using single top tchannel cross section and $(t \rightarrow W b) /(t \rightarrow W q)$ fraction measurements. The $\Gamma_{\text {top }}$ is calculated with quantum mechanical relation, $\Gamma_{\text {top }}=\frac{\sigma(t-c h)}{B r(t \rightarrow b W)} \cdot \frac{B r(t \rightarrow b W)_{\mathrm{SM}}}{\sigma(t-c h)_{\mathrm{SM}}}$. The result, $\Gamma_{\text {top }}=2.00_{-0.43}^{+0.47} \mathrm{GeV}$, is the most precise determination of the top quark width using experimental data sample and consistent with the SM [17].

\subsection{W boson helicity}

The SM predicts that the top quark decays almost entirely to a $W$ boson and a bottom quark, and that the $W t b$ vertex is a V-A charged weak current interaction [2]. A consequence of this is that approximately $70 \%$ of the top quark decay longitudinally, $30 \%$ of the top quarks have left handed polarization $\left(\mathrm{f}_{0}=70 \%, \mathrm{f}_{-}=30 \%, \mathrm{f}_{+}=0 \%\right)$ [18]. Any new particles involved in the same decay topologies and non-standard coupling could create a different mixture of polarized $W$ bosons. Therefore, a measurement of this fraction is a test of the V-A nature of the $W t b$ vertex. D0 collaboration uses both lepton+jets and dilepton channel simultaneously with $4.3 \mathrm{fb}^{-1}$ data and extracts $\mathrm{f}_{+}=0.02 \pm 0.05$ and $\mathrm{f}_{0}=0.67 \pm 0.10$ with the simultaneous fit of the two variables [19]. CDF collaboration has results in both lepton+jets [20] and dilepton channels [21]

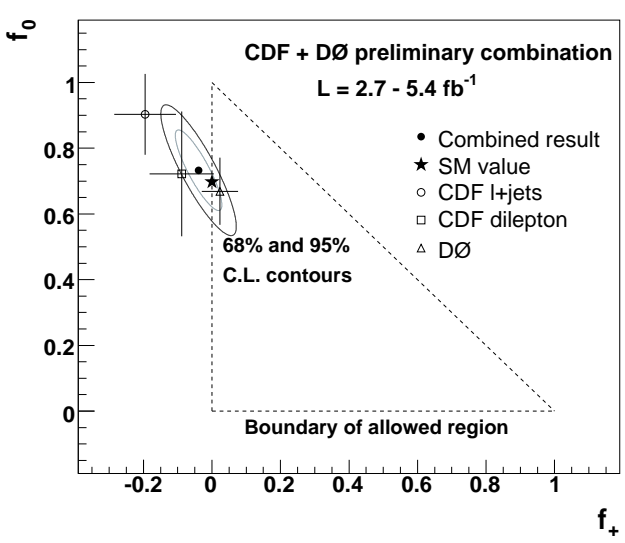

Figure 4. Combined tevatron result on $\mathrm{W}$ boson helicity measurement with 1 and 2 standard deviation contours are compared with the SM prediction.

with partial data of the Run II. All three different measurements are combined to get $\mathrm{f}_{+}=-0.013 \pm 0.035$ and $\mathrm{f}_{0}=0.685 \pm 0.057$ [22] which is consistent with the SM prediction as one can see in Fig. 4. CDF collaboration update the measurement in the lepton+jets channel with full data set corresponding to an integrated luminosity of $8.7 \mathrm{fb}^{-1} p \bar{p}$ collisions and obtain $\mathrm{f}_{+}=-0.045 \pm 0.043$ and $\mathrm{f}_{0}=0.726 \pm 0.067$ [23] which is also consistent with the $\mathrm{SM}$ prediction. This result can improve the combination of Tevatron measurements.

\subsection{The radio $B(t \rightarrow W b) / B(t \rightarrow W q)$}

In the SM, top quark decay almost exclusively into a $W$ boson and a bottom quark with an assumption of a three generation quark-mixing matrix [2]. The existence of a fourth generation of quarks would remove this constraint and allow the decay into the other down type quarks such as $d$ and $s$ quarks. Therefore, the measurement of the branching fraction ratios $R=\frac{B(t \rightarrow W b)}{B(t \rightarrow W q)}$ is sensitive to the new physics. The decay rate of the top quark into a $W$ boson and a down-type quark $q(q=d s, b)$ is proportional to $\left|V_{t b}\right|^{2}$, the square element of the Cabibbo-KobayashiMaskawa (CKM) matrix. D0 collaboration uses data from $5.4 \mathrm{fb}^{-1}$ of $p \bar{p}$ collisions in both lepton + jets and dilepton channels. The $R$ can be extracted with number of $b$-tagged jets as shown in Fig. 5 (up) and measures $R=0.90 \pm 0.04$, and extract the $\left|V_{t b}\right|=0.95 \pm 0.02$ assuming three generation of quarks [24]. CDF collaboration has a measurement in the lepton+jets channel with full data set of $8.7 \mathrm{fb}^{-1}$. The jet multiplicities and the number of $b$-tagged jets as shown in Fig. 5 (down) are used to extract $R=0.94 \pm 0.09$. We also extract the $\left|V_{t b}\right|=0.97 \pm 0.05$ with the assumption of three generation quark mixing [25]. The measured $V_{t b}$ are approximately 2 standard deviation from the SM prediction. However, it is still consistent. 


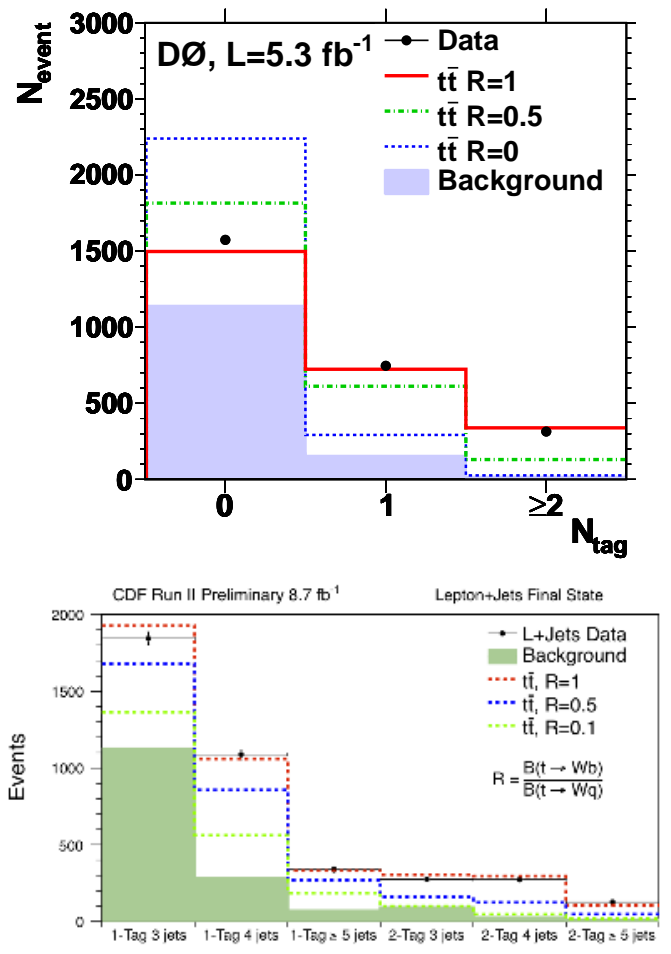

Figure 5. Up: Number of $b$-tagged jets distribution from the D0 $5.4 \mathrm{fb}^{-1}$ data in the lepton+jets channel are compared with various predictions from different $R$. Down: Number of jets and $b$-tagging of the CDF $8.7 \mathrm{fb}^{-1}$ data in the lepton+jets channel are compared with various predictions from different $R$.

\section{Conclusion}

The CDF and D0 collaborations have performed a robust set of analyses using many techniques and improvements to have better understanding the top quark nature. With Tevatron shutdown at last year, we have $>10 \mathrm{fb}^{-1}$ of data acquired in each experiment. Many new measurements use the full data set but, still lots of ongoing measurements are underway with increasing data set as well as improving machinery.

\section{Acknowledgments}

I would like to thank for the CDF and D0 colleagues for their efforts to carry out these challenging physics analyses. I also thank for the conference organizers for a very rich week of physics. My travel was supported by the National Research Foundation of Korea Grant funded by the Korean Government [NRF-2011-35B-C00007].

\section{References}

[1] F. Abe el al. (CDF Collaboration), Phys. Rev. Lett. 74, 2626 (1995); S. Abachi el al. (D0 Collaboration), Phys. Rev. Lett. 74, 2632 (1995).

[2] K. Nakamura el al. (Particle Data Group), J. Phys. G 37, 075021 (2010).

[3] ALEPH, CDF, D0, DELPHI, L3, OPAL, SLD, the LEP Electroweak Working Group, the Tevatron Electroweak Working Group, and the SLD Electroweak and Heavy Flavor Working Groups, arXiv:1012.2367v2.

[4] H. Flächer el al., Eur. Phys. J. C 60, 543 (2009).

[5] T. Aaltonen el al. (CDF and D0 Collaborations), Phys. Rev. D 86, 092003 (2012).

[6] T. Aaltonen el al. (CDF Collaboration), Phys. Rev. Lett. 109, 152003 (2012).

[7] V. Abazov (D0 Collaboration), Phys. Rev. D 86, 051103 (2012).

[8] CDF Collaboration, CDF conference note 10810.

[9] A. H. Hoang and J. W. Stewart, Nucl. Phys. Proc. Suppl. 185, 220 (2008).

[10] U. Langenfeld, S. Moch, and P. Uwer, Phys. Rev. D 80, 054009 (2009).

[11] A. H. Hoang, A. Jain, I. Scimemi, and I. W. Stewart, Phys. Rev. Lett. 101, 151602 (2008).

[12] V. Abazov et al. (D0 Collaboration), Phys. Lett. B 703, 422 (2011).

[13] V. Abazov el al. (D0 Collaboration), Phys. Rev. Lett. 103, 13200 (2009); V. Abazov el al. (D0 Collaboration), Phys. Rev. D 84, 052005 (2011).

[14] T. Aaltonen el al. (CDF Collaboration), Phys. Rev. Lett. 106, 152001 (2011); T. Aaltonen el al. (CDF Collaboration), arXiv:1210.6131.

[15] G. J. Feldman and R. D. Cousins, Phys. Rev. D 57, 3873 (1998).

[16] (CDF Collaboration), CDF conference note 10936.

[17] V. Abazov el al. (D0 Collaboration), Phys. Rev. D 85, 091104 (2012).

[18] J. Aguilar-Saavedra el al., Eur. Phys. J. C 50, 519 (2007).

[19] V. Abazov el al. (D0 Collaboration), Phys. Rev. D 83, 032009 (2011).

[20] T. Aaltonen el al. (CDF Collaboration), Phys. Rev. Lett. 105, 042002 (2010).

[21] T. Aaltonen el al. (CDF Collaboration), arXiv:1205.0354.

[22] T. Aaltonen el al. (CDF and D0 Collaborations), Phys. Rev. D 85, 071106 (2012).

[23] T. Aaltonen el al. (CDF Collaboration), arXiv:1211.4523.

[24] V. Abazov el al. (D0 Collaboration), Phys. Rev. Lett. 107, 121802 (2011).

[25] (CDF Collaboration), CDF conference note 10887. 